\title{
A Retrospective Study of Noninvasive Prenatal Testing for Chromosome Aneuploidies and Sub-Chromosomal Copy Number Variations in 24359 Single Pregnancies
}

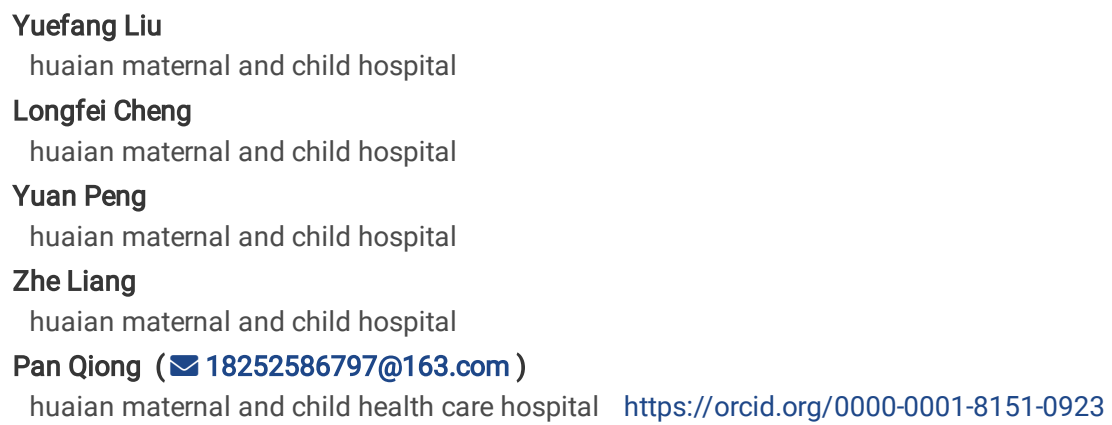




\section{Abstract}

Background: With the development of whole-genome sequencing, small sub-chromosomal deletions and duplications could be found by non-invasive prenatal testing(NIPT). This study aimed to review the efficiency of NIPT as a screening test for aneuploidies and sub-chromosomal copy number variations (CNVs) in 24359 single pregnancies.

Methods: A total of 24359 single pregnancies with different clinical indications were retrospectively analyzed. The positive predictive value $\triangle \mathrm{PPV} \llbracket$ of chromosome aneuploidies and subchromosomal CNVs were analyzed. Pathogenicity of abnormal NIPT results were assessed according to American College of Medical Genetics and Genomics(ACMG).

Results: A total of 442 pregnancies (442/24359,1.9\%) were with abnormal NIPT results. PPV for trisomy 21(T21), trisomy 18 (T18), trisomy 13 (T13), and sex chromosome aneuploidies (SCAs) was $84.8 \%, 54.2 \%, 11.1 \%$ an $40.5 \%$ respectively. The PPV for sub-chromosomal CNVs was 59.0\% (46/78). The PPV for CNVs $\leq 5 \mathrm{Mb}$ was $68.9 \%$ (31/45), for CNVs within 5-10 Mb was $83.3 \%(5 / 6)$ and for CNVs $\geq 10 \mathrm{Mb}$ was $37.1 \%$ (10/27) respectively. The clinical information, prenatal diagnosis results and follow-up results of 46 true positive cases, 6 cases with sub-chromosomal CNVs inconsistent with NIPT and 1 false negative case were also described in detail.

Conclusions: Our data have potential significance in demonstrating the significance of NIPT not only for common whole chromosome aneuploidies but also for sub-chromosomal CNVs. Besides, the clinical information, prenatal diagnosis results and follow-up results of 52 cases with sub-chromosomal CNVs and 1 false negative case would provide important guidance for genetic counseling.

\section{Introduction}

The discovery of cell-free fetal DNA (cffDNA) in maternal plasma by Lo et al in 1997 has opened up new approaches for NIPT[1]. At present, NIPT has been gradually applied as a first-tier aneuploidy screening strategy in clinical practice [2-3]. Previous large-scale clinical studies have revealed high accuracy of NIPT in screening on trisomy 21,18 and 13 , with sensitivity and specificity higher than $95 \%$ [4-5].

Genomic structural changes, such as copy number variations (CNVs), are also always associated with human disease. Recently, further development and expansion use of NIPT has focused on microduplication/microdeletion syndromes(MMSs)[6]. The most common microdeletion is at 22q11.2, and recent reports indicate that the clinical incidence rate may exceed 1/1000 in the prenatal population[7]. The 22q11.2 microdeletion syndrome causing DiGeorge syndrome has a very broad clinical phenotype that can include congenital heart disease, palatal, gastrointestinal, genitourinary anomalies, immunodeficiency, endocrine disturbance, developmental delay, cognitive deficits and psychiatric illness[8]. In addition to 22q11.2 microduplication/microdeletion syndromes, other newly described CNVs like the distal 1q21.1 microdeletions/microduplications, 15q11.2 deletion, 16p13.11 deletion and 16p11.2 deletion/duplication are also identified as disease-causing CNVs[9-12]. Therefore, it is very important to evaluate the accuracy of NIPT for CNVs, which could help identify high-risk pregnancies and offer the possibility of a confirmatory invasive diagnostic test. However, there are many challenges for NIPT in clinical practice especially low positive predictive values (PPV). In the present study, we retrospectively analyzed 24359 single pregnancies including 125 cases of sub-chromosomal CNVs to assess the efficiency of NIPT technology on the detection of sub-chromosomal CNVs.

\section{Results}

\section{An overview of clinical data}

Among the 24367 cases undergoing NIPT, 8 cases were not eligible for the next analysis due to the low concentration of fetal DNA, so the remaining 24359 cases were under analyzed in the present study. The maternal age for the 24359 pregnancies ranged from 16 to 51 years old. The group aged 25 to 29 years was the majority $(8084,33.2 \%)$. Pregnant women older than 35 years were 3966(16.3\%). The gestational age ranged from 9 to 34 weeks, and $56.9 \%$ had a gestational age from 17 to 20 weeks (see Table 1). The positive rate from younger group to older group were 1.4\%(85/6097), 1.8\%(144/8084), 1.5\%(95/6212), $2.6 \%(92 / 3472)$ and 5.3\%(26/494) respectively. (Fig. 1). Older group ( $\geq 41)$ has the highest positive rate. 
Table 1

Maternal age and gestational age of 24359 blood sampling

\begin{tabular}{|lll|}
\hline Maternal age at NIPT (years) & Number & Percent (100\%) \\
\hline$\leq 24$ & 6097 & $25.0 \%$ \\
\hline $25-29$ & 8084 & $33.2 \%$ \\
\hline $30-34$ & 6212 & $25.5 \%$ \\
\hline $35-40$ & 3472 & $14.3 \%$ \\
\hline$\geq 41$ & 494 & $2.0 \%$ \\
\hline Advanced maternal age ( $\geq 35$ years old) & 3966 & $16.3 \%$ \\
\hline Gestational age at NIPT (weeks) & & \\
\hline$\leq 8$ & 0 & $0 \%$ \\
\hline $9-12$ & 680 & $2.8 \%$ \\
\hline $13-16$ & 6525 & $26.8 \%$ \\
\hline $17-20$ & 13867 & $56.9 \%$ \\
\hline $21-24$ & 2612 & $10.7 \%$ \\
\hline $25-28$ & 639 & $2.6 \%$ \\
\hline$\geq 29$ & 36 & $0.1 \%$ \\
\hline Range (weeks) & $12-34$ & $/$ \\
\hline
\end{tabular}

Figure 1 The positive rate of NIPT for aneuploidy and subchromosomal CNV increases with maternal age and the number of positive cases in the five age groups.

There were 442 pregnant cases with chromosome aneuploidies and submicroscopic anomalies, whose basic information and clinical reasons for NIPT were collected (Table 2 and Table 3). We found that 61(13.8\%,61/442) cases were with advanced maternal age more than 35 years (included), and $26(5.8 \%$, $26 / 442)$ pregnant cases had ultrasound abnormalities. $123(27.8 \%, 123 / 442)$ cases were with high risk of serum biochemistry screening. The number of the voluntary group were $210(47.5 \%, 210 / 442)$. Other reasons included poor fertility history and maternal chromosomal abnormality or mental retardation.

Table 2

Clinical information of 442 pregnancy cases with chromosome aneuploidies and subchromosomal CNVs

\begin{tabular}{|lll|}
\hline Maternal age at NIPT (years) & Number & Percent (100\%) \\
\hline$\leq 24$ & 77 & $17.4 \%$ \\
\hline $25-29$ & 148 & $33.9 \%$ \\
\hline $30-34$ & 95 & $21.5 \%$ \\
\hline $35-40$ & 97 & $21.9 \%$ \\
\hline$\geq 41$ & 25 & $5.7 \%$ \\
\hline Advanced maternal age ( $\geq 35$ years old) & 122 & $27.6 \%$ \\
\hline Gestational age at NIPT (weeks) & & \\
\hline$\leq 8$ & 0 & $0 \%$ \\
\hline $9-12$ & 13 & $2.9 \%$ \\
\hline $13-16$ & 116 & $26.2 \%$ \\
\hline $17-20$ & 252 & $57.0 \%$ \\
\hline $21-24$ & 46 & $10.4 \%$ \\
\hline $25-28$ & 13 & $2.9 \%$ \\
\hline$\geq 29$ & 2 & $0.5 \%$ \\
\hline Range (weeks) & $12-30^{+1}$ & \\
\hline
\end{tabular}


Table 3

Reasons of 442 pregnant cases with chromosome aneuploidies and subchromosomal CNVs

\begin{tabular}{|lll|}
\hline Reasons & Number & Percent (100\%) \\
\hline Advanced age & 61 & $13.8 \%$ \\
\hline Ultrasound abnormalities & 26 & $5.9 \%$ \\
\hline Abnormal serum biochemistry screening & 123 & $27.8 \%$ \\
\hline Poor fertility histroy & 2 & $0.5 \%$ \\
\hline Voluntary detection & 210 & $47.5 \%$ \\
\hline
\end{tabular}

NIPT results for T21, T18, T13 and sex chromosome aneuploidies

In 442 pregnancies with abnormal NIPT, there were 12 cases of T13, 35 cases of T18, 90 cases of T21, and 106 cases of sex chromosome abnormalities (SCAs) (Table 4). Among them, there were 102 true-positive cases, 76 false-positive cases, and 65 unverified cases that chose to continue gestation or to terminate the pregnancy. For the 102 true-positive cases, there were 56 cases of T21, 1 case of T13, 13 cases of T18, and 32 cases of SCAs (Table 4).

Table 4

PPV of common chromosome aneuploidie

\begin{tabular}{|c|c|c|c|c|c|c|}
\hline Common chromosome aneuploidie & Cases & Unverified & $\begin{array}{l}\text { prenatal } \\
\text { diagnosis }\end{array}$ & $\begin{array}{l}\text { True } \\
\text { Positive }\end{array}$ & $\begin{array}{l}\text { False } \\
\text { positive }\end{array}$ & PPV \\
\hline $\mathrm{T} 21$ & 90 & 24 & 66 & 56 & 10 & $84.8 \%$ \\
\hline T18 & 35 & 11 & 24 & 13 & 11 & $54.2 \%$ \\
\hline T13 & 12 & 3 & 9 & 1 & 8 & $11.1 \%$ \\
\hline SCAs & 106 & 27 & 79 & 32 & 47 & $40.5 \%$ \\
\hline Total & 243 & 65 & 178 & 102 & 76 & $57.3 \%$ \\
\hline
\end{tabular}

\section{Nipt Results For Other Chromosome Aneuploidie}

In 442 pregnancies with abnormal NIPT results, there were 74 cases of other chromosome aneuploidie including 39 cases of T7, 9 cases of T20, 12 case of T16, 3 cases of T10, 6 cases of T8, 3 cases of T9 and 2 cases of T14. Only 43 cases chosen amniocentesis for further diagnosis and result showed that there was only one true positive cases in T20. Therefore, the PPV for other chromosome aneuploidie in our study was $1.3 \%$.

Table 5

Different PPVs according to pregnancies characteristics

\begin{tabular}{|llllll|}
\hline Indications & PPV of T21 & PPV of T18 & PPV of T13 & PPV of SCAs & PPV of CNVs \\
\hline Fetal structural abnormalities by ultrasound & 0 & $100 \%(1 / 1)$ & 0 & 0 & 0 \\
\hline Abnormal soft index of ultrasound & $50.0 \%(1 / 2)$ & $0(0 / 1)$ & 0 & $16.7 \%(1 / 6)$ & $60.0 \%(3 / 5)$ \\
\hline Abnormal serological screening & $75.0 \%(24 / 32)$ & $55.6 \%(5 / 9)$ & $20.0 \%(1 / 5)$ & $36.7 \%(11 / 30)$ & $62.1 \%(18 / 29)$ \\
\hline Advanced maternal age ( $\geq 35$ years) & $80.0 \%(24 / 30)$ & $60.0 \%(6 / 10)$ & $0 \%(0 / 2)$ & $50.0 \%(11 / 22)$ & $46.2 \%(6 / 13)$ \\
\hline Other & 0 & 0 & 0 & $25.0 \%(1 / 4)$ & $71.4 \%(5 / 7)$ \\
\hline No clinical indications & $25.0 \%(1 / 4)$ & $33.3 \%(1 / 3)$ & $0(0 / 2)$ & $37.5 \%(6 / 16)$ & $53.6 \%(15 / 28)$ \\
\hline
\end{tabular}

\section{Nipt Results For Cnvs}

In addition, NIPT could also identify positive cases of sub-chromosomal CNVs. In 442 pregnancies with abnormal NIPT, there were 125 (28.3\%,125/442) cases of sub-chromosomal CNVs. After genetic counseling about the clinical significance of these sub-chromosomal CNVs, 78 cases, including 27 cases with subchromosomal $C N V \geq 10 \mathrm{M}, 45$ cases with $\mathrm{CNV} \leq 5 \mathrm{M}$ and 6 cases with CNV within 5-10 Mb, chosen to perform amniotic fluid puncture for further prenatal diagnosis. The PPV for sub-chromosomal CNVs screened by NIPT were $59 \%$ (46/78). The PPV for CNVs $\leq 5 \mathrm{Mb}$ was $68.9 \%$ (31/45), for CNVs within $5-10 \mathrm{Mb}$ was $83.3 \%(5 / 6)$ and for $\mathrm{CNVs} \geq 10 \mathrm{Mb}$ was $37.1 \%$ (10/27) respectively. Remaining 32 false positive cases included 6 cases with inconsistent CNV and 26 cases with no abnormality. Among 52 abnormal cases, 31 cases were correlated to microdeletion or microduplication syndromes suggesting that NIPT may be an important method to find potential birth defect. Moreover, we compared fetal free DNA concentration between 46 true positive cases and 32 false positive cases. The result showed that true positive cases had higher fetal fraction than false positive cases $(p=0.013)($ Fig. 2$)$. The clinical information, prenatal diagnosis results and follow-up results of 52 abnormal cases are shown in the Table 6 and Table 7 (sorted by CNV size) 
Table 6

The clinical information, prenatal diagnosis results and follow-up results of 15 cases with CNVs $\geq 10 \mathrm{M}$.

\begin{tabular}{|c|c|c|c|c|c|c|c|c|c|}
\hline No & age & $\begin{array}{l}\text { Preg- } \\
\text { week }\end{array}$ & Indication & NIPT & Karyotype & Chip & siginificance & origin & $\overline{F_{1}}$ \\
\hline 1 & 38 & $\begin{array}{l}26+ \\
0\end{array}$ & $\begin{array}{l}\text { advanced } \\
\text { age }\end{array}$ & $\begin{array}{l}\text { dup of3q24- } \\
\text { q29(146M- } \\
194 \mathrm{M})\end{array}$ & $\begin{array}{l}46, X X, \operatorname{der}(8) t(3 ; 8) \\
\text { (q24;p23) }\end{array}$ & $\begin{array}{l}3 q 24 q 29(144,804,358- \\
197,851,444) \times 3,8 p 23.3 p 23.1(158,048- \\
6,982,257) \times 1\end{array}$ & Pathogenic & & Tt \\
\hline 2 & 26 & 19 & voluntary & $\begin{array}{l}\text { dup of13q14.11- } \\
\text { q21.32 } \\
(46-65 M)\end{array}$ & $\begin{array}{l}\text { 46,XN,dup (13) } \\
\text { (q14q21) }\end{array}$ & $\begin{array}{l}13 q 14.11 q 21.32(43,450,607- \\
66,726,903) \times 3\end{array}$ & Pathogenic & farther & $T_{\epsilon}$ \\
\hline 3 & 23 & $\begin{array}{l}17+ \\
2\end{array}$ & voluntary & $\begin{array}{l}\text { dup of } \\
\text { 18p11.32- } \\
\text { p11.21 } \\
(1 \mathrm{M}-11 \mathrm{M}) \text {, }\end{array}$ & normal & $15 q 11.2(22,754,322-23,222,284) \times 1$ & $\begin{array}{l}\text { Uncertain } \\
\text { significance }\end{array}$ & unknown & ur \\
\hline 4 & 24 & $\begin{array}{l}17+ \\
2\end{array}$ & voluntary & $\begin{array}{l}\text { dup } \\
\text { of } 4 q 24.3(1(19 M- \\
144 M)\end{array}$ & normal & $4 p 16.3 p 16.1(71,566-9,371,116) \times 1$ & Pathogenic & De novo & Tt \\
\hline 5 & 30 & $\begin{array}{l}20+ \\
6\end{array}$ & voluntary & $\begin{array}{l}\text { del ofXq23-q28 } \\
(110 M-153 M)\end{array}$ & $46, X, \operatorname{del}(X)(q 23)$ & $\begin{array}{l}\text { Xp22.31(6,386,248- } \\
8,141,017) \times 3 ; X q 23 q 28(110,798,069- \\
152,651,757) \times 1\end{array}$ & $\begin{array}{l}\text { Uncertain } \\
\text { significance }\end{array}$ & unknown & $\begin{array}{l}N \\
f \in\end{array}$ \\
\hline 6 & 31 & $\begin{array}{l}17+ \\
6\end{array}$ & voluntary & $\begin{array}{l}\text { dup of19q13.2- } \\
\text { q13.43 } \\
(42 M-57 M)\end{array}$ & normal & $\begin{array}{l}\text { 11p14.1p13(27,473,981 - } \\
33,896,715) \times 2 \text { hmz }\end{array}$ & $\begin{array}{l}\text { Uncertain } \\
\text { significance }\end{array}$ & unknown & ur \\
\hline 7 & 30 & $\begin{array}{l}17+ \\
6\end{array}$ & $\begin{array}{l}\text { High risk } \\
\text { of serum } \\
\text { screening }\end{array}$ & $\begin{array}{l}\text { del of } 5 \mathrm{p} 15.33- \\
\text { p13.3 } \\
(1 \mathrm{M}-29 \mathrm{M})\end{array}$ & $\begin{array}{l}46, \mathrm{XN}, \mathrm{del}(5) \\
\text { (p14.2p15.3) }\end{array}$ & $5 p 15.33 p 14.2(38,139-23,389,253) \times 1$ & Pathogenic & unknown & al \\
\hline 8 & 33 & 18 & $\begin{array}{l}\text { High risk } \\
\text { of serum } \\
\text { screening }\end{array}$ & $\begin{array}{l}\text { del of } 5 \mathrm{p} 15.33- \\
\text { p15.2 } \\
(15 \mathrm{M})\end{array}$ & $46, \mathrm{XN}, \mathrm{del}(5)(\mathrm{p} 15.1)$ & 5p15.33p15.1(113,576 - 16,275,896)x1 & $\begin{array}{l}\text { Pathogenic(cri- } \\
\text { du-chat } \\
\text { syndrome) }\end{array}$ & De novo & Tt \\
\hline 9 & 22 & $\begin{array}{l}19+ \\
4\end{array}$ & $\begin{array}{l}\text { High risk } \\
\text { of serum } \\
\text { screening }\end{array}$ & $\begin{array}{l}\text { del of } 5 q 14.3 \\
(48 M)\end{array}$ & none & $\begin{array}{l}5 q 14.3 q 23.2(82,812,442- \\
121,787,549) \times 1\end{array}$ & Pathogenic & unknown & Tt \\
\hline 10 & 24 & 22 & voluntary & $\begin{array}{l}\text { dup of } 2 q 33.1- \\
\text { q37.3 }(199 \mathrm{M}- \\
241 \mathrm{M})\end{array}$ & $\begin{array}{l}\text { 46,XN,dup (2) } \\
\text { (q33.1q37.1) }\end{array}$ & none & Pathogenic & unknown & $T_{\epsilon}$ \\
\hline 11 & 25 & $\begin{array}{l}13+ \\
4\end{array}$ & voluntary & $\begin{array}{l}\text { del of11q12.1- } \\
\text { q13.2(56M-67M) }\end{array}$ & normal & $16 q 23.1(77,909,692-78,568,430) \times 3$ & Pathogenic & unknown & ne \\
\hline 12 & 38 & 16 & voluntary & $\begin{array}{l}\text { dup of3q27.1- } \\
\text { q29 } \\
(183-196 \mathrm{M})\end{array}$ & none & $\begin{array}{l}\text { 4.8M del of } 5 q 35.2-5 q 35.3 \\
13.1 \mathrm{M} \text { dup of } 3 q 27.2-3 q 29\end{array}$ & $\begin{array}{l}5 \mathrm{q} 35 \mathrm{del} \\
\text { syndrome }\end{array}$ & De novo & $\begin{array}{l}\mathrm{Ft} \\
\mathrm{cc} \\
\mathrm{m}\end{array}$ \\
\hline 13 & 24 & 19 & $\begin{array}{l}\text { High risk } \\
\text { of serum } \\
\text { screening }\end{array}$ & $\begin{array}{l}\text { dup of16q13.3- } \\
13.11 \\
(1-26 M) \\
\text { dup of } 16 q 12.2- \\
24.3 \\
(49-88 M)\end{array}$ & normal & $16 p 12.2(21,966,869-22,662,193) \times 1$ & $\begin{array}{l}\text { Uncertain } \\
\text { significance }\end{array}$ & mother & ne \\
\hline 14 & 27 & $\begin{array}{l}19+ \\
4\end{array}$ & $\begin{array}{l}\text { High risk } \\
\text { of serum } \\
\text { screening }\end{array}$ & $\begin{array}{l}\text { dup of10p15.3- } \\
\text { p12.2 } \\
(1 \mathrm{M}-23 \mathrm{M}) \\
\text { del of } \\
12 \mathrm{q} 13.33- \\
\text { q13.32 } \\
(1 \mathrm{M}-5 \mathrm{M})\end{array}$ & $\begin{array}{l}\text { 46,XN,der(12)t(10;12) } \\
\text { (p12.31;p13.31) }\end{array}$ & $\begin{array}{l}\text { 12p13.33p13.31(173,786- } \\
6,437,099) \times 1,10 p 15.3 p 12.31(100,047- \\
20,255,943) \times 3\end{array}$ & Pathogenic & unknown & Tt \\
\hline
\end{tabular}




\begin{tabular}{|c|c|c|c|c|c|c|c|c|c|}
\hline No & age & $\begin{array}{l}\text { Preg- } \\
\text { week }\end{array}$ & Indication & NIPT & Karyotype & Chip & siginificance & origin & $F_{1}$ \\
\hline 15 & 24 & $2^{18+}$ & $\begin{array}{l}\text { High risk } \\
\text { of serum } \\
\text { screening }\end{array}$ & $\begin{array}{l}\text { del of } \\
8 p 23.2-23.3 \\
(1 M-4 M), \\
\text { dup of } \\
21 q 21.3-22.3 \\
(28 M-46 M)\end{array}$ & $\begin{array}{l}46, \mathrm{XN}, \text { der(8)t(8;21) } \\
\text { (23.2; } 21.3)\end{array}$ & $\begin{array}{l}\text { 8p23.3p23.2(158,048- } \\
4,896,398) \times 1,21 q 21.3 q 22.3(27,985,829 \\
-48,093,361) \times 3\end{array}$ & Pathogenic & unknown & Tt \\
\hline
\end{tabular}


Table 7

The clinical information, prenatal diagnosis results and follow-up results of 37 cases with CNV $<10 \mathrm{M}$ and 1 false negative I

\begin{tabular}{|c|c|c|c|c|c|c|c|c|}
\hline No & age & $\begin{array}{l}\text { Preg } \\
\text { week }\end{array}$ & Indication & NIPT & Karyotype & Chip & siginificance & origin \\
\hline 16 & 29 & $\begin{array}{l}18+ \\
5\end{array}$ & $\begin{array}{l}\text { Abnormal } \\
\text { ultrasound }\end{array}$ & $\begin{array}{l}\text { del of } 5 \mathrm{p} 14.1- \\
\text { p13.3(26M- } \\
29 \mathrm{M})\end{array}$ & normal & $\begin{array}{l}\text { 5p14.1(26,067,126- } \\
28,837,434) \times 1,17 \mathrm{p} 13.3(716,837- \\
1,201,192) \times 3\end{array}$ & Uncertain significance & unknown \\
\hline 17 & 24 & $\begin{array}{l}16+ \\
1\end{array}$ & voluntary & $\begin{array}{l}\text { dup of } 16 \mathrm{p} 12.2 \\
(22 \mathrm{M}-23 \mathrm{M})\end{array}$ & normal & $\begin{array}{l}\text { 16p12.2(21,841,353 - } \\
22,431,031) \times 3\end{array}$ & Uncertain significance & unknown \\
\hline 18 & 27 & $\begin{array}{l}17+ \\
0\end{array}$ & voluntary & $\begin{array}{l}\text { del of } 15 q 13.1- \\
\text { q14 } \\
(30 M-34 M)\end{array}$ & normal & $\begin{array}{l}15 q 13.2 q 13.3(30,955,149- \\
32,513,176) \times 1\end{array}$ & $15 q 13.3$ del syndrome & mother \\
\hline 19 & 28 & $\begin{array}{l}17+ \\
2\end{array}$ & voluntary & $\begin{array}{l}\text { dup of } \\
\text { Xq21.33-q22.3 } \\
(98 M-107 M)\end{array}$ & normal & $\begin{array}{l}\text { Xq21.33q22.3(97,776,700 - } \\
107,811,504) \times 3\end{array}$ & $\begin{array}{l}\text { PathogenicPelizaeus- } \\
\text { Merzbacher disease }\end{array}$ & mother \\
\hline 20 & 29 & $\begin{array}{l}17+ \\
2\end{array}$ & voluntary & $\begin{array}{l}\text { del of } 17 \mathrm{p} 13.3- \\
\text { p13.2 } \\
(0.1 \mathrm{M}-6 \mathrm{M})\end{array}$ & normal & 17p13.3p13.2(4,888-4,818,558)x1 & $\begin{array}{l}\text { miller- } \\
\text { diekerlissencephaly } \\
\text { syndrome }\end{array}$ & unknown \\
\hline 21 & 29 & $\begin{array}{l}14+ \\
6\end{array}$ & voluntary & $\begin{array}{l}\text { dup of } 8 \mathrm{p} 23.3- \\
\text { p23.2 } \\
(1 \mathrm{M}-5 \mathrm{M})\end{array}$ & normal & $8 p 23.2(3,687,399-5,950,104) \times 4$ & uncertain significance & unknown \\
\hline 22 & 31 & $\begin{array}{l}19+ \\
4\end{array}$ & voluntary & $\begin{array}{l}\text { del of } 17 p 13.3 \\
(0.1 M-3 M)\end{array}$ & normal & $\begin{array}{l}\text { 17p13.3(1,330,366- } \\
3,059,811) \times 1 ; 17 q 12(34,824,845- \\
36,339,294) \times 3\end{array}$ & $\begin{array}{l}\text { miller- } \\
\text { diekerlissencephaly } \\
\text { syndrome }\end{array}$ & unknown \\
\hline 23 & 34 & $\begin{array}{l}21+ \\
0\end{array}$ & voluntary & $\begin{array}{l}\text { del of } \\
16 \mathrm{p} 13.12- \\
\text { p12.3(15M- } \\
17 \mathrm{M})\end{array}$ & normal & $\begin{array}{l}\text { 16p13.12p13.11(14,780,640- } \\
16,458,424) \times 1\end{array}$ & $\begin{array}{l}\text { 16p13.11 recurrent } \\
\text { region (includes } \\
\text { MYH11) }\end{array}$ & unknown \\
\hline 24 & 28 & $\begin{array}{l}16+ \\
6\end{array}$ & $\begin{array}{l}\text { High risk } \\
\text { of serum } \\
\text { screening }\end{array}$ & $\begin{array}{l}\text { dup of } \\
22 q 11.21 \\
(18.9 \mathrm{M}-22 \mathrm{M},)\end{array}$ & $46, X N$,inv(9)p11q13 & $\begin{array}{l}3 p 26.3(1,536,945- \\
2,579,649) \times 3 ; 22 q 11.21(18,844,632 \\
-21,462,353) \times 3\end{array}$ & $\begin{array}{l}\text { 22q11.2 dup } \\
\text { syndrome }\end{array}$ & unknown \\
\hline 25 & 33 & $\begin{array}{l}19+ \\
3\end{array}$ & $\begin{array}{l}\text { High risk } \\
\text { of serum } \\
\text { screening }\end{array}$ & $\begin{array}{l}\text { del of Xp11.23- } \\
\text { p11.22 } \\
(48-53 \mathrm{M})\end{array}$ & normal & $\begin{array}{l}\text { Xp11.23p11.22(48,735,882- } \\
53,521,570) \times 1\end{array}$ & uncertain significance & unknown \\
\hline 26 & 24 & $\begin{array}{l}18+ \\
6\end{array}$ & $\begin{array}{l}\text { High risk } \\
\text { of serum } \\
\text { screening }\end{array}$ & $\begin{array}{l}\text { dup of } 21 \mathrm{q} 21.1 \\
(17 \mathrm{M}-18 \mathrm{M})\end{array}$ & normal & $\begin{array}{l}21 q 21.1(17,775,056- \\
19,154,417) \times 3\end{array}$ & uncertain significance & unknown \\
\hline 27 & 32 & $\begin{array}{l}17+ \\
0\end{array}$ & $\begin{array}{l}\text { mild risk } \\
\text { of serum } \\
\text { screening }\end{array}$ & $\begin{array}{l}\text { dup of } \\
15 q 11.2-q 13.1 \\
((23 M-28 M,)\end{array}$ & normal & $\begin{array}{l}15 q 11.2 q 13.1(22,764,491- \\
29,071,810) \times 3\end{array}$ & $\begin{array}{l}15 q 11-q 13 \text { dup } \\
\text { syndrome }\end{array}$ & unknown \\
\hline 28 & 25 & $\begin{array}{l}17+ \\
0\end{array}$ & $\begin{array}{l}\text { mild risk } \\
\text { of serum } \\
\text { screening }\end{array}$ & $\begin{array}{l}\text { del of } 4 \text { q34.1- } \\
\text { q34.3 } \\
(182 \mathrm{M}-183 \mathrm{M})\end{array}$ & normal & $\begin{array}{l}\text { 4q34.3q35.1(182,542,070- } \\
183,305,274) \times 1\end{array}$ & uncertain significance & unknown \\
\hline 29 & 25 & $3^{17+}$ & $\begin{array}{l}\text { mild risk } \\
\text { of serum } \\
\text { screening }\end{array}$ & $\begin{array}{l}\text { dup of } \\
22 \mathrm{q} 11.21(19 \mathrm{M}- \\
20 \mathrm{M})\end{array}$ & normal & $\begin{array}{l}22 q 11.21(18,648,855- \\
21,800,471) \times 3\end{array}$ & $\begin{array}{l}\text { 22q11.2 dup } \\
\text { syndrome }\end{array}$ & unknown \\
\hline 30 & 31 & $\begin{array}{l}20+ \\
0\end{array}$ & $\begin{array}{l}\text { mild risk } \\
\text { of serum } \\
\text { screening }\end{array}$ & $\begin{array}{l}\text { dup of } \\
22 \mathrm{q} 11.21- \\
\text { q11.23 } \\
(22 \mathrm{M}-24 \mathrm{M})\end{array}$ & normal & $\begin{array}{l}22 q 11.21 q 11.23(21,059,669- \\
24,629,406) \times 3\end{array}$ & $\begin{array}{l}22 q 11.2 \text { recurrent } \\
\text { region (distal type I, D- } \\
\text { E/F) }\end{array}$ & unknown \\
\hline 31 & 22 & $\begin{array}{l}17+ \\
5\end{array}$ & $\begin{array}{l}\text { mild risk } \\
\text { of serum } \\
\text { screening }\end{array}$ & $\begin{array}{l}\text { dup of17q12 } \\
(35 \mathrm{M}-37 \mathrm{M})\end{array}$ & normal & $17 q 12(34,822,465-36,243,365) \times 3$ & $17 q 12$ dup syndrome & mother \\
\hline 32 & 27 & $\begin{array}{l}24+ \\
5\end{array}$ & $\begin{array}{l}\text { Widening } \\
\text { of lateral } \\
\text { ventricle }\end{array}$ & $\begin{array}{l}\text { dup of } \\
13 \mathrm{q} 12.11- \\
\text { q12.12 } \\
(22 \mathrm{M}-24 \mathrm{M})\end{array}$ & none & $\begin{array}{l}13 q 12.12(23,554,650- \\
24,826,638) \times 3\end{array}$ & uncertain significance & unknown \\
\hline
\end{tabular}




\begin{tabular}{|c|c|c|c|c|c|c|c|c|}
\hline No & age & $\begin{array}{l}\text { Preg } \\
\text { week }\end{array}$ & Indication & NIPT & Karyotype & Chip & siginificance & origin \\
\hline \multirow[t]{2}{*}{33} & 25 & 22 & voluntary & $\begin{array}{l}\text { del of } \\
\text { Xq27.1q27.3 }\end{array}$ & none & $\begin{array}{l}\text { Xq27.1q27.3(138,661,694- } \\
143,597,022) \times 1\end{array}$ & uncertain significance & unknown \\
\hline & & & & $(137 \mathrm{M}-143 \mathrm{M})$ & & & & \\
\hline \multirow[t]{2}{*}{34} & 31 & 19 & $\begin{array}{l}\text { Maternal } \\
\text { mental } \\
\text { retardation }\end{array}$ & $\begin{array}{l}\text { dup of } \\
22 q 12.1 q 12.2\end{array}$ & none & $\begin{array}{l}\text { 22q12.1q12.2(28,317,927 - } \\
30,826,759) \times 2 \mathrm{hmz}\end{array}$ & uncertain significance & $\begin{array}{l}\text { Mother- } \\
\text { 22q11.23(23, }\end{array}$ \\
\hline & & & & $(23 M-24 M)$ & & & & $25,086,816) x_{i}^{\prime}$ \\
\hline \multirow[t]{2}{*}{35} & 22 & $26+$ & $\begin{array}{l}\text { Maternal } \\
\text { cleft lip }\end{array}$ & $\begin{array}{l}\text { del of } \\
22 q 11.21\end{array}$ & none & $\begin{array}{l}22 q 11.21(18,648,855- \\
21,800,471) \times 1\end{array}$ & DiGeorge syndrome & unknown \\
\hline & & & and palate & $(18 M-21 M)$ & & & & \\
\hline 36 & 29 & $\begin{array}{l}23+ \\
5\end{array}$ & voluntary & $\begin{array}{l}\text { del of 5p15.33- } \\
\text { p15.2 (0M-3M) }\end{array}$ & none & $5 \mathrm{p} 15.33(38,139-2,436,105) \times 1$ & $\begin{array}{l}\text { CRI-DU-CHAT } \\
\text { syndrome }\end{array}$ & De novo \\
\hline \multirow[t]{2}{*}{37} & 32 & $\begin{array}{l}17+ \\
2\end{array}$ & voluntary & $\begin{array}{l}\text { dup of } \\
22 q 11.21- \\
\text { q11.22 }\end{array}$ & none & $\begin{array}{l}22 q 11.21 q 11.23(21,464,120- \\
23,650,987) \times 3\end{array}$ & $\begin{array}{l}22 q 11.2 \text { dup } \\
\text { syndrome }\end{array}$ & unknown \\
\hline & & & & $(21 \mathrm{M}-23 \mathrm{M})$ & & & & \\
\hline \multirow[t]{2}{*}{38} & 27 & $16+$ & mild risk & del of $5 q 12.1$ & none & $5 q 12.1(59,052,591-60,864,744) \times 1$ & uncertain significance & mother \\
\hline & & & screening & $(59 M-61 M)$ & & & & \\
\hline \multirow[t]{2}{*}{39} & 27 & 17 & $\begin{array}{l}\text { high risk } \\
\text { of serum }\end{array}$ & $\begin{array}{l}\text { dup of } \\
10 q 23.1-23.2\end{array}$ & none & $\begin{array}{l}10 q 22.3-23.2(81,674,866- \\
88,970,446) \times 3\end{array}$ & likely pathogenic & De novo \\
\hline & & & screenıng & $(82-89 \mathrm{M})$ & & & & \\
\hline \multirow[t]{2}{*}{40} & 27 & $\begin{array}{l}25+ \\
4\end{array}$ & $\begin{array}{l}\text { Increased } \\
\text { bowel }\end{array}$ & $\begin{array}{l}\text { del of } 15 q 11.2- \\
\text { q12 }\end{array}$ & normal & $\begin{array}{l}15 q 11.2 q 13.1(23,300,172- \\
28,536,634) \times 1\end{array}$ & $\begin{array}{l}\text { angelman syndrome! } \\
\text { prader-Willi syndrome }\end{array}$ & De novo \\
\hline & & & $\begin{array}{l}\text { widened } \\
\text { lateral } \\
\text { ventricle }\end{array}$ & $(24-27 M)$ & & & & \\
\hline \multirow[t]{2}{*}{41} & 27 & 21 & voluntary & $\begin{array}{l}\text { dup of 3q12.3- } \\
\text { q13.11 }\end{array}$ & normal & $\begin{array}{l}3 q 12.3 q 13.11(101,694,516- \\
104,402,138) \times 3\end{array}$ & uncertain significance & mother \\
\hline & & & & $(101-104 M)$ & & & & \\
\hline \multirow[t]{2}{*}{42} & 24 & $23^{+3}$ & $\begin{array}{l}\text { mild risk } \\
\text { of serum }\end{array}$ & $\begin{array}{l}\text { dup of } 17 \mathrm{p} 12- \\
\text { p11.2 }\end{array}$ & none & $17 p 12(14,099,504-15,424,086) \times 3$ & $\begin{array}{l}\text { 17p12 recurrent } \\
(\mathrm{HNPP} / \mathrm{CMT} 1 \mathrm{~A})\end{array}$ & mother \\
\hline & & & screening & $(14-16 \mathrm{M})$ & & & $\begin{array}{l}\text { region (includes } \\
\text { PMP22) }\end{array}$ & \\
\hline \multirow[t]{2}{*}{43} & 23 & 23 & $\begin{array}{l}\text { Maternal } \\
\text { mental }\end{array}$ & del of $17 q 12$ & normal & $17 q 12(34,822,465-36,410,720) \times 1$ & $17 q 12$ recurrent del & Mother(menté \\
\hline & & & retardation & $(34-36 \mathrm{M})$ & & & & \\
\hline \multirow[t]{2}{*}{44} & 39 & $\begin{array}{l}15+ \\
55\end{array}$ & $\begin{array}{l}\text { History of } \\
\text { adverse }\end{array}$ & $\begin{array}{l}\text { del of } 4 q 12- \\
\text { q13.1 }\end{array}$ & none & $4 q 12(52,920,475-59,495,539) \times 1$ & likely pathogenic & De novo \\
\hline & & & gna & $(53-62 M)$ & & & & \\
\hline \multirow[t]{2}{*}{45} & 35 & $13+$ & voluntary & dup of $17 p 12$ & normal & $3 q 28(189,409,398-$ & 17p12 recurrent & unknown \\
\hline & & & & $(14 \mathrm{M}-16 \mathrm{M})$ & & $-15,413,862) \times 3$ & & \\
\hline 46 & 33 & 18 & $\begin{array}{l}\text { high risk } \\
\text { of serum } \\
\text { screening }\end{array}$ & $\begin{array}{l}\text { del of Xp22.32- } \\
\text { p22.31(6M- } \\
8 M)\end{array}$ & none & Xp22.31 $(6,455,151-8,141,076) \times 1$ & pathogenic & mother \\
\hline \multirow[t]{2}{*}{47} & \multirow[t]{2}{*}{25} & \multirow[t]{2}{*}{20} & \multirow{2}{*}{$\begin{array}{l}\text { mild risk } \\
\text { of serum } \\
\text { screening }\end{array}$} & $\begin{array}{l}\text { dup of } 1 \mathrm{p} 13.2- \\
\text { p12 }\end{array}$ & none & $\begin{array}{l}1 \mathrm{p} 13.2 \mathrm{p} 12(115,582,990- \\
120,527,348) \times 3\end{array}$ & uncertain significance & $\begin{array}{l}\text { 15q11.2-motl } \\
\text { 1p13.2p12-de }\end{array}$ \\
\hline & & & & $(116-119 M)$ & & $\begin{array}{l}\text { 15q11.2(22,770,421 - } \\
23,276,605) \times 1\end{array}$ & & \\
\hline 48 & 29 & 20 & $\begin{array}{l}\text { Maternal } \\
\text { mental } \\
\text { retardation }\end{array}$ & $\begin{array}{l}\text { del of Xq27.2- } \\
\text { q27.3(143M- } \\
147 M)\end{array}$ & none & $\begin{array}{l}\text { Xq27.3q28(142,954,184 - } \\
147,171,818) \times 0\end{array}$ & likely pathogenic & unknown \\
\hline 49 & 35 & $\begin{array}{l}15+ \\
6\end{array}$ & $\begin{array}{l}\text { Advanced } \\
\text { age }\end{array}$ & $\begin{array}{l}\text { del of 9p21.3- } \\
\text { p21.1(25M- } \\
30 M)\end{array}$ & none & $\begin{array}{l}9 p 21.3 p 21.1(24,796,507- \\
30,288,265) \times 1\end{array}$ & uncertain significance & mother \\
\hline \multirow[t]{2}{*}{50} & 28 & $22+$ & high risk & dup of $7 q 32.3$ & none & 4q33q34.3(170,186,543 - & uncertain significance & unknown \\
\hline & & & screening & $(132 \mathrm{M}-135 \mathrm{M})$ & & & & \\
\hline
\end{tabular}




\begin{tabular}{|c|c|c|c|c|c|c|c|c|}
\hline No & age & $\begin{array}{l}\text { Preg } \\
\text { week }\end{array}$ & Indication & NIPT & Karyotype & Chip & siginificance & origin \\
\hline 51 & 35 & 22 & voluntary & $\begin{array}{l}\text { del of Xq24q25 } \\
(117-122 M)\end{array}$ & none & $\begin{array}{l}\text { Xq24q25(117,865,893 - } \\
122,724,000) \times 1\end{array}$ & uncertain significance & unknown \\
\hline 52 & 30 & 12 & voluntary & $\begin{array}{l}\text { dup of } 8 p 23.2 \\
(3-5 M)\end{array}$ & none & $8 p 23.2(3,687,399-5,950,104) \times 4$ & uncertain significance & unknown \\
\hline 53 & 28 & $20+$ & $\begin{array}{l}\text { High risk } \\
\text { of serum } \\
\text { screening }\end{array}$ & low risk & 46,XX,del(4)(p14) & none & pathogenic & De novo \\
\hline
\end{tabular}

Figure 2 Fetal free DNA fraction of NIPT between true positive group $(X \pm S=17.3 \pm 5.7, n=46)$ and false positive group $(X \pm S=14.0 \pm 7.6, n=32)(p=0.0185)$.

\section{Different Ppv According To Pregnancies Characteristics}

We also analyzed different PPV according to pregnancies characteristics, Different PPVs of NIPT according to pregnancies indications are shown in Table 5. The total PPV of T21 was $84.8 \%$, the PPV of T21 fetuses in women of advanced maternal age was $80.0 \%$ and in abnormal serological screening group was $75.0 \%$. Similarly, the PPV of T18 and SCAs in advanced maternal age group were also the highest. It is worth noting that PPVs of CNVs in pregnancies with cleft lip, mental retardation or history of bad pregnancy was the highest.

\section{Five Cases With Distal 22q11.2 Microdeletions And Microduplications}

In our study, the PPV of 22q11.2 microdeletions and microduplications is $100 \%$ (5/5). There were four fetuses with $22 q 11.2$ duplication syndrome and one with 22q11.2 deletion syndrome (Table 8). Case 24, 29 and 37 were all clinically healthy after birth but case 30 was with ventricular septal defect, aortic abnormalities and 1 bright spot in the left ventricle subsequently detected by ultrasound. Case 35 was confirmed to have a $22 q 11.21$ deletion syndrome with hoarseness, atrial septal defect, patent ductus arteriosus and myocardial injury after birth. However, it was not clear that whether these mutations were de novo or inherited from parents. Case 34 was confirmed to have the loss of heterozygosity in 22q12.1q12.2 whose clinical phenotypes were unavailable. Fetal mother were also advised to have a array comparative genomic hybridization because of her mental retardation. CMA analysis showed that $22 \mathrm{q} 11.23$ microduplication may contribute to her mental retardation.

Table 8

The clinical information, prenatal diagnosis results and follow-up results of six cases distal 22q11.2 microdeletions and microduplications.

\begin{tabular}{|c|c|c|c|c|c|c|c|}
\hline No & age & $\begin{array}{l}\text { Pregnant } \\
\text { week }\end{array}$ & $22 q$ Dup/del & $\begin{array}{l}\text { bp start; stop } \\
\text { (NCBI37/hg19) }\end{array}$ & significance & origin & Follow-up \\
\hline 24 & 28 & $16+6$ & duplication & $\begin{array}{l}3 p 26.3(1,536,945- \\
2,579,649) \times 3 ; 22 q 11.21(18,844,632 \\
-21,462,353) \times 3\end{array}$ & $\begin{array}{l}\text { 22q11.2 dup } \\
\text { syndrome }\end{array}$ & unknown & normal \\
\hline 29 & 25 & $17+3$ & duplication & $\begin{array}{l}22 q 11.21(18,648,855- \\
21,800,471) \times 3\end{array}$ & $\begin{array}{l}\text { 22q11.2 dup } \\
\text { syndrome }\end{array}$ & unknown & normal \\
\hline 30 & 31 & 20 & duplication & $\begin{array}{l}22 q 11.21 q 11.23(21,059,669- \\
24,629,406) \times 3\end{array}$ & $\begin{array}{l}22 \mathrm{q} 11.2 \\
\text { recurrent region } \\
\text { (distal type I, D- } \\
\text { E/F) }\end{array}$ & unknown & $\begin{array}{l}\text { congenital heart } \\
\text { malformation,ventricular } \\
\text { septal defect, aortic } \\
\text { abnormalities }\end{array}$ \\
\hline 34 & 31 & & $\begin{array}{l}\text { loss of } \\
\text { heterozygosity }\end{array}$ & $\begin{array}{l}\text { 22q12.1q12.2(28,317,927 - } \\
30,826,759) \times 2 \mathrm{hmz}\end{array}$ & $\begin{array}{l}\text { uncertain } \\
\text { significance }\end{array}$ & $\begin{array}{l}\text { mother with } \\
22 q 11.23(23,700,639 \\
-25,086,816) \times 3\end{array}$ & unknown \\
\hline 35 & 22 & $26+2$ & deletion & $\begin{array}{l}22 q 11.21(18,648,855- \\
21,800,471) \times 1\end{array}$ & $\begin{array}{l}\text { DiGeorge } \\
\text { syndrome }\end{array}$ & unknown & $\begin{array}{l}\text { hoarseness, congenital } \\
\text { heart disease }\end{array}$ \\
\hline 37 & 32 & $17+2$ & duplication & $\begin{array}{l}22 q 11.21 q 11.23(21,464,120- \\
23,650,987) \times 3\end{array}$ & $\begin{array}{l}22 q 11.2 \text { dup } \\
\text { syndrome }\end{array}$ & unknown & normal \\
\hline
\end{tabular}

\section{One Case Of Xp22.31 Microdeletion}

Case 46

was detected to have a 1.6 Mb microdeletion in Xp22.31 by NIPT, which often causes ichthyosis (X-linked recessive genetic disease). Most female carriers of Xp22.31 microdeletion have a normal phenotype, a few female carriers may show abnormal symptoms due to inactivation of $X$ chromosomes and all male carriers show ichthyosis. In our study, case 46

chosen to perform prenatal diagnosis for further confirmation and the result showed that the fetus was female and this mutation is inherited from her normal mother. 


\section{One Case Of 5.4 mb Microdeletion In 9p21.3-p21.1}

\section{Case 49}

was detected to have a 5.4 Mb microdeletion in 9p21.3-p21.1(24,796,507-30,288,265) by NIPT, which contains 8 OMIM genes such as TEK and C9orf72. Heterozygous mutations of TEK gene often causes venous malformations, multiple cutaneous and mucosal (autosomal dominant genetic disease). C9orf72 is related with frontotemporal dementia and/or amyotrophic lateral sclerosis 1 (autosomal dominant genetic disease). However, case 49

was healthy and this $5.4 \mathrm{Mb}$ microdeletion was inherited from his/her healthy mother. Interestingly, our laboratory had previously detected a $4.2 \mathrm{Mb}$ microdeletion in 9p21.2p21.1(26,210,360 - 30,492,812) by CMA from a fetus with NT $=1.3 \mathrm{~cm}$ and this $4.2 \mathrm{Mb}$ microdeletion was de novo. These results suggested that 9p21.3-p21.1 microdeletion may have penetrance difference.

\section{A false negative NIPT result for case 53 with 4 p14 microdeletion}

The NIPT result of case 53 with high risk of serum screening was normal. System structure screening (other hospital) in 24 week showed fetal growth retardation. The fetal was diagnosed as neonatal pneumonia, low weight, congenital heart disease and hyperbilirubinemia after birth. Karyotype detection of peripheral blood showed $46, \mathrm{XX}$, del(4)(p4). Thus, case 53 was tested again by improved the experimental method with better cffDNA enrichment. The results showed increased cffDNA fraction from 6.5-15.1\% and a 34Mbp deletion in 4p16.3-p15.1 region, which is co-related with Wolf-Hirschhorn syndrome (WHS).

\section{Discussion}

In this study, we are the first to reviewed the efficiency of NIPT for screening common chromosome aneuploidies as well as sub-chromosomal CNV within a cohort of 24359 single pregnancies in Huaian area. This NIPT technology uses a semiconductor sequencing platform with high enrichment of cffDNA (20\%-40\%) to reliably detect subchromosomal deletions/duplications. The PPV for T21, T18, T13 and SCAs was $84.8 \%, 54.2 \%, 11.1 \%$ an $40.5 \%$ respectively. In several recent studies, the PPV of T21 was $65-94 \%$, the PPV of T18 was $47-85 \%$, and the PPV of T13 was $12-62 \%$ [13-15]. Our results are consistent with previous studies. Interestingly, the PPV for CNVs was 59.0\%, which is obviously higher than previous studies with 9-36\% [16-18]. The reason for higher PPV of CNVs in our study may be related with our new enrichment strategy. The PPV for CNVs $\leq 5 \mathrm{Mb}$ was $68.9 \%$ (31/45), for CNVs within 5-10 Mb was 83.3\% $(5 / 6)$ and for CNVs $\geq 10 \mathrm{Mb}$ was $37.1 \%$ (10/27) respectively. However, previous reports demonstrated that PPV for CNVs $\geq 10 \mathrm{Mb}$ was significantly higher than CNVs $<10 \mathrm{Mb}$ [16-18]. Further analysis showed that there were only 27 cases with big CNVs ( $\geq 10 \mathrm{Mb}$ ) but 51 cases with small CNVs ( $10 \mathrm{Mb}$ ) suggesting that small CNVs occurred more frequently than big CNVs. Therefore, we speculate that frequent occurrence of small CNVs may be the potential cause of higher PPV of small CNVs.

Further analysis about the different PPV of NIPT according to pregnancies indications was performed. The results showed that the PPV of NIPT was the highest for T21 and was much lower for other aneuploidies. PPV of CNVs was close to T18 and much higher than T13. Advanced maternal age is a high risk factor for T21 so PPV of T21 in advanced maternal age the highest. PPV of CNVs in advanced maternal age group was lower suggesting that advanced maternal age was not significantly related with CNVs. PPVs of CNVs in pregnancies with cleft lip, mental retardation or history of bad pregnancy was the highest suggesting high risk factors for CNVs.

Among the 46 true positive cases and 7 abnormal false positive cases, 31 cases were correlated to microdeletion or microduplication syndromes with 6 cases inherited from parents, 3 cases de novo and other 22 cases unavailable. Early detection of pathogenic and potentially pathogenic CNVs by NIPT has good benefit in prenatal screening. 22q11.2 microduplication was the most frequent in our research. The phenotype of the five patients with $22 q 11.2$ microduplications were diverse, with symptoms ranging from being normal to mental retardation and congenital heart malformation. $22 q 11.2$ microdeletions is the second most common chromosomal abnormality secondary to Down syndrome [19]. However, the occurrence of $22 q 11.2$ microduplications was more frequent than 22q11.2 microdeletion in our study, which was contrary to previous research conclusion. The rare occurrence of $22 q 11.2$ microduplication cases may be explained by the absence of a defined phenotype and incomplete penetrance[20].

Studies have demonstrated that there is a small chance of a false negative result for NIPT[21]. In our study, there was a false negative case in 79 validated NIPT. The most common factor associated with these false negative results is the low fetal fraction, which are often affected by maternal weight, gestational age and extraction method[22-23]. In our research, extraction method for cffDNA enrichment was the main reason for the false negative cases. Therefore, improved extraction method for elevating fetal fraction were immediately used in December 2018, which may be the potential reason for improved the overall performance of NIPT and higher PPV in this research. Faas BH et al in 2012 clarified that cell free fetal DNA in the maternal plasma originates from cytotrophoblastic cells derived from trophoblast of the blastocyst[24]. The karyotypes of cytotrophoblast and fetus may be different due to fetus are derived from the inner cell mass (ICM) of the blastocyst[21]. Other reasons for false negative results may be CPM and maternal mosaicism[25-27].

\section{Conclusions}

This study demonstrated that the PPV for T21, T18, T13 and SCAs was $85 \%, 54 \%, 11 \%$ and $41 \%$ respectively. The PPV for CNVs was $59.0 \%$. The PPV for CNVs $\leq 5 \mathrm{Mb}$ was $68.9 \%$ (31/45), for CNVs within 5-10 Mb was $83.3 \%(5 / 6)$ and for CNVs $\geq 10 \mathrm{Mb}$ was $37.1 \%$ (10/27) respectively. Our data have potential significance in demonstrating the usefulness of NIPT not only for common whole chromosome aneuploidies but also for CNVs.

\section{Materials And Methods}

\section{Patients}


From 2015 to July 2019, 24359 pregnant women opted for NIPT to screen fetal chromosome aneuploidies. Informed written consent was obtained from all pregnant women who agreed to receive NIPT. Pregnancies with high risks were divided into advanced maternal age, ultrasound abnormalities, poor fertility history, positive serum screening, and other groups in this study.

\section{NIPT sequencing}

Maternal peripheral blood $(5 \mathrm{ml})$ was collected in an ethylenediaminetetraacetic acid (EDTA) tube. The blood sample was stored at $4{ }^{\circ} \mathrm{C}$ immediately after collection. Afterwards, cfDNA extraction, library construction, quality control, and pooling were performed according to the JingXin Fetal Chromosome Aneuploidy (T21, T18, T13) Testing Kits (CFDA registration permit No. 0153400300). Sequencing reads were filtered and aligned to the human reference genome (hg19). Combined GC correction and Z-score testing methods were used to identify fetal autosomal aneuploidies. A cut off value of Z-score > 3 was used to determine whether the ratio of the chromosomes was increased. Here, each chromosome with an absolute value of the Z-score greater than 3 was

marked with chromosome aneuploidies or microdeletions/ microduplications.

\section{Chromosome karyotype analysis}

Banding cytogenetics was performed on G-banded metaphase chromosomes of cultured peripheral blood lymphocytes using routine techniques. Karyotypes were interpreted according to the International System for Human Cytogenetic Nomenclature.

\section{Chromosome karyotype analysis}

The aCGH platform from CytoScanTM 750 k chip made by American Affymetrix was employed in this study. The standard operating procedures for genomic DNA digestion, ligation, amplification, purification, fragmentation, labeling, chip hybridization, washing and scanning, and data analysis were performed. Pathogenicity of genomic DNA fragments were determined with reference to the international public pathological CNVs database, international public pathological CNVs database, international public benign CNVs database and human genetics cytogenetics microarray database.

\section{Abbreviations}

NIPT: Noninvasive prenatal testing; cffDNA: Cell-free DNA; CMA: Chromosomal microarray analysis; CNVs: Copy number variants; MMS:

Microdeletion/microduplication syndromes; PPV: Positive predictive value; LCRs: Low copy repeats; NAHR: Non-allelic homologous recombination; CPM: Confined placental mosaicism; ICM: Inner cell mass.

\section{Declarations}

\section{Ethics approval and consent to participate}

This study was approved by the Ethics Committee of Huaian Maternal and Child Health Care Hospital.

\section{Consent for publication}

The authors declare that they have no competing interests and the patients in this case report had provided their consent for publication.

\section{Availability of data and materials}

The datasets used and/or analyzed during the current study are available from the corresponding author on reasonable request.

\section{Competing interests}

The authors declare that they have no competing interests.

Funding: Supported by the Maternal and Child Health project of Jiangsu Province (No. F201670هF201714) (Pan Qiong)区the "333 Project" Foundation of Jiangsu Province (No. BRA2017250) (Pan Qiong).

\section{Authors'contributions}

All authors have materially participated in the study and manuscript preparation. YF Liu, LF Chen, Y Peng, Z Liang, X Jin and NN Yan collected all clinical data. YF Liu participated in the data analysis and drafted the manuscript. Q Pan designed the work and drafted and revised the manuscript. All authors have approved the final article.

\section{Acknowledgements}

We would like express our gratitude for financial support from Maternal and Child Health project of Jiangsu Province (No. F201670खF201714) and the "333 Project" Foundation of Jiangsu Province (No. BRA2017250).

\section{References}

1. Lo YM, Corbetta N, Chamberlain PF, Rai V, Sargent IL, Redman CW, et al. Presence of fetal DNA in maternal plasma and serum. Lancet. 1997;350(9076):485-7.

2. Filoche S, Lawton B, Beard A, Dowell A, Stone P. New screen on the block: non-invasive prenatal testing for fetal chromosomal abnormalities. J Prim Health Care. 2017;9(4):248-53. 
3. McCullough RM, Almasri EA, Guan X, Geis JA, Hicks SC, Mazloom AR, et al. Non-invasive prenatal chromosomal aneuploidy testing-clinical experience: 100,000 clinical samples. PLoS One. 2014;9(10):e109173.

4. Hu H, Liu H, Peng C, Deng T, Fu X, Chung C, et al. Clinical Experience of Non-Invasive Prenatal Chromosomal Aneuploidy Testing in 190,277 Patient Samples. Curr Mol Med. 2016;16(8):759-66.

5. Liang D, Lin Y, Qiao F, Li H, Wang Y, Zhang J, et al. Perinatal outcomes following cell-free DNA screening in > 32000 women: Clinical follow-up data from a single tertiary center. Prenat Diagn. 2018;38(10):755-64.

6. Oskarsdottir S, Vujic M, Fasth A. Incidence and prevalence of the 22q11 deletion syndrome: a population-based study in Western Sweden. Arch Dis Child. 2004;89(2):148-51.

7. Grati FR, Molina GD, Ferreira JC, Dupont C, Alesi V, Gouas L, et al. Prevalence of recurrent pathogenic microdeletions and microduplications in over 9500 pregnancies.2015; 35(8):801-809.

8. McDonald-McGinn DM, Sullivan KE, Marino B, Philip N, Swillen A, Vorstman JA, et al. 22q11.2 deletion syndrome. Nat Rev Dis Primers.2015.

9. Qiao Y, Badduke C, Tang F, Cowieson D, Martell S, Lewis SME, et al. Whole exome sequencing of families with 1q21.1 microdeletion or microduplication. Am J Med Genet A. 2017;173(7):1782-91.

10. Cox DM, Butler MG. The 15q11.2 BP1-BP2 microdeletion syndrome: a review. Int J Mol Sci. 2015;16(2):4068-82.

11. Smith AE, Jnah A, Newberry D. Chromosome 16p13.11 Microdeletion Syndrome in a Newborn: A Case Study. Neonatal Netw. 2018;37(5):303-9.

12. Rosenfeld JA, Coe BP, Eichler EE, Cuckle H, Shaffer LG. Estimates of penetrance for recurrent pathogenic copy-number variations. Genet Med. 2013;15(6):478-81.

13. Neofytou MC, Tsangaras K, Kypri E, Loizides C, loannides M, Achilleos A, et al. Targeted capture enrichment assay for non-invasive prenatal testing of large and small size sub-chromosomal deletions and duplications. PLoS One. 2017;12(2):e0171319.

14. Yaron Y, Jani J, Schmid M, Oepkes D. Current Status of Testing for Microdeletion Syndromes and Rare Autosomal Trisomies Using Cell-Free DNA Technology. Obstet Gynecol. 2015;126(5):1095-9.

15. Sentilhes L, Salomon LJ, Vayssiere C. Cell-free DNA Analysis for Noninvasive Examination of Trisomy. N Engl J Med. 2015;373(26):2581-2.

16. Chen Y, Yu Q, Mao X, Lei W, He M, Lu W. Noninvasive prenatal testing for chromosome aneuploidies and subchromosomal microdeletions/microduplications in a cohort of 42,910 single pregnancies with different clinical features. Hum Genomics. 2019;13(1):60.

17. Hu H, Wang L, Wu J, Zhou P, Fu J, Sun J, et al. Noninvasive prenatal testing for chromosome aneuploidies and subchromosomal microdeletions/microduplications in a cohort of 8141 single pregnancies. Hum Genomics. 2019;13(1):14.

18. Liang D, Cram DS, Tan H, Linpeng S, Liu Y, Sun H, et al. Clinical utility of noninvasive prenatal screening for expanded chromosome disease syndromes. Genet Med. 2019;21(9):1998-2006.

19. McDonald-McGinn DM, Tonnesen MK, Laufer-Cahana A, Finucane B, Driscoll DA, Emanuel BS, et al. Phenotype of the 22q11.2 deletion in individuals identified through an affected relative: cast a wide FISHing net! Genet Med. 2001;3(1):23-9.

20. Wincent J, Bruno DL, van Bon BW, Bremer A, Stewart H, Bongers EM, et al. Sixteen New Cases Contributing to the Characterization of Patients with Distal 22q11.2 Microduplications. Mol Syndromol. 2010;1(5):246-54.

21. Bianchi DW, Wilkins-Haug L. Integration of noninvasive DNA testing for aneuploidy into prenatal care: what has happened since the rubber met the road? Clin Chem. 2014;60(1):78-87.

22. Ashoor G, Syngelaki A, Poon LC, Rezende JC, Nicolaides KH. Fetal fraction in maternal plasma cell-free DNA at 11-13 weeks' gestation: relation to maternal and fetal characteristics. Ultrasound Obstet Gynecol. 2013;41(1):26-32.

23. Jorgez CJ, Dang DD, Simpson JL, Lewis DE, Bischoff FZ. Quantity versus quality: optimal methods for cell-free DNA isolation from plasma of pregnant women. Genet Med. 2006;8(10):615-9.

24. Faas BH, de Ligt J, Janssen I, Eggink AJ, Wijnberger LD, van Vugt JM, et al. Non-invasive prenatal diagnosis of fetal aneuploidies using massively parallel sequencing-by-ligation and evidence that cell-free fetal DNA in the maternal plasma originates from cytotrophoblastic cells. Expert Opin Biol Ther.

2012;12(Suppl 1):19-26.

25. Jiang F, Ren J, Chen F, Zhou Y, Xie J, Dan S, et al. Noninvasive Fetal Trisomy (NIFTY) test: an advanced noninvasive prenatal diagnosis methodology for fetal autosomal and sex chromosomal aneuploidies. BMC Med Genomics.2012; 5(57.

26. Kalousek DK. Pathogenesis of chromosomal mosaicism and its effect on early human development. Am J Med Genet. 2000;91(1):39-45.

27. Gao Y, Stejskal D, Jiang F, Wang W. False-negative trisomy 18 non-invasive prenatal test result due to $48, \mathrm{XXX},+18$ placental mosaicism. Ultrasound Obstet Gynecol. 2014;43(4):477-8.

\section{Figures}




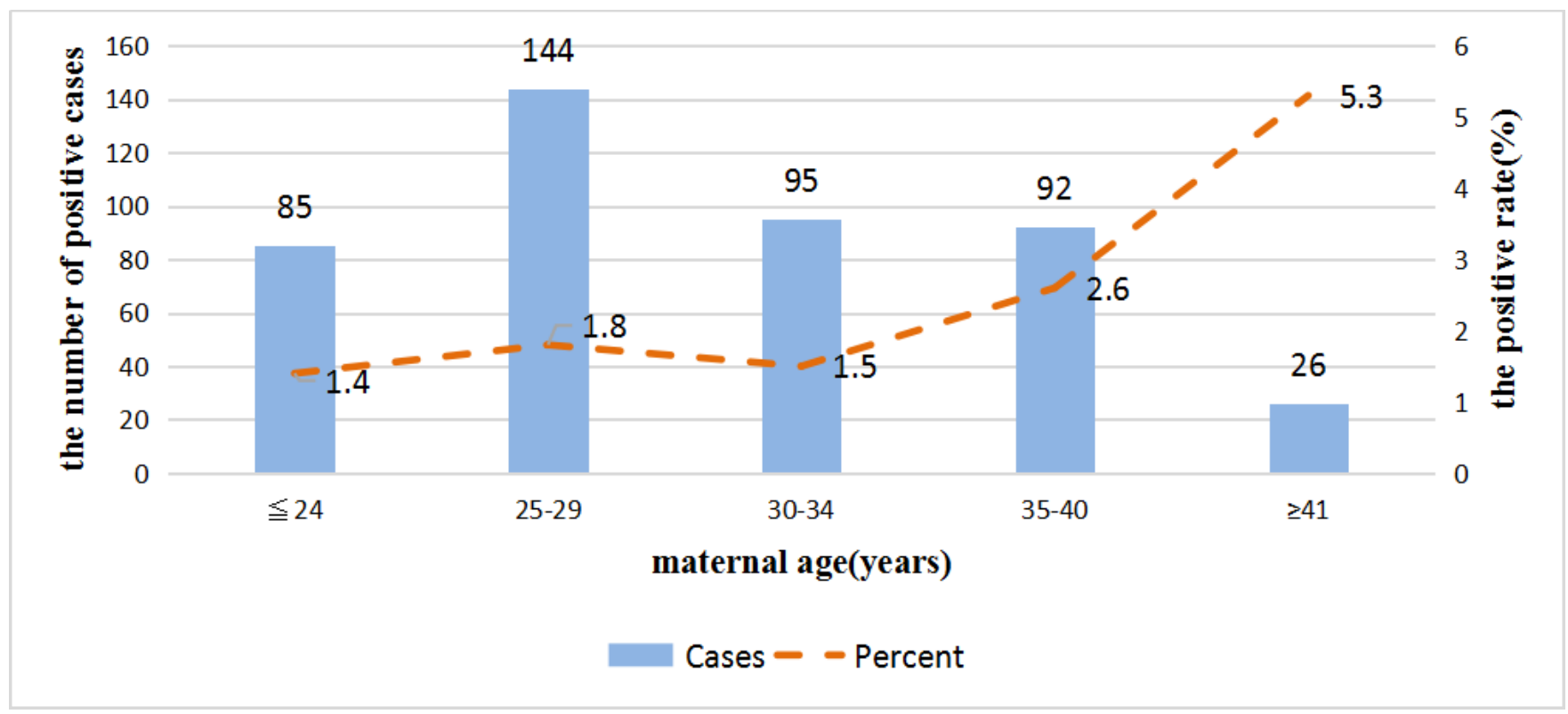

Figure 1

The positive rate of NIPT for aneuploidy and subchromosomal CNV increases with maternal age and the number of positive cases in the five age groups.

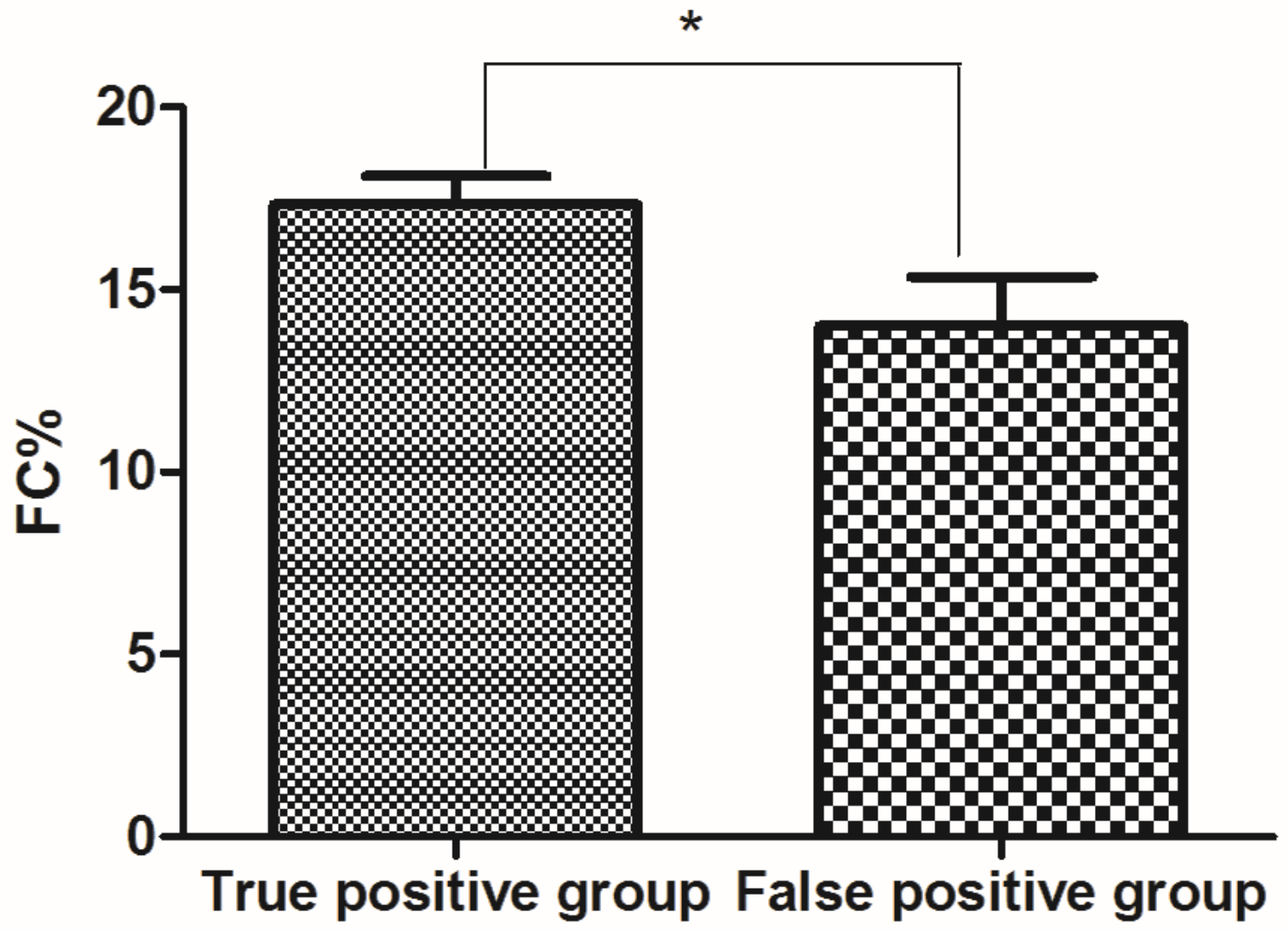

Figure 2

Fetal free DNA fraction of NIPT between true positive group $(X \pm S=17.3 \pm 5.7, n=46)$ and false positive group $(X \pm S=14.0 \pm 7.6, n=32)(p=0.0185)$. 